\title{
The Actual Nature of Light ( I ) Reveal the Mystery about the Actual Nature of Light from Newton, Einstein to the Recent Mistakes
}

\author{
He-Zhou Wang*, He-Xiang He, Jie Feng, Xiao-Dong Chen, Wei Lin \\ State Key Laboratory of Optoelectronic Materials and Technologies, Zhongshan University, Guangzhou, China \\ E-mail: ${ }^{*}$ stswhz@mail.sysu.edu.cn \\ Received June 3, 2011; revised June 30, 2011; accepted July 10, 2011
}

\begin{abstract}
When Newton became the President of the Royal Society, he proposed corpuscle concept (wave-particle duality) to destroy the fruitions of Hooke and Huygens, because Newton mistook Hooke and Huygens as his enemies. Thereafter, this erroneous concept governed the scientific world for more than one hundred years. This paper will reveal the mystery: why corpuscle concept could govern the scientific world for one hundred years after Newton's death. In the beginning of last century, photon, a palingenesis of Newton's corpuscle, was proposed by Einstein again, as a sudden whim, because Planck strongly opposed this wrong concept, since 1907, Einstein strongly doubted this concept. Finally, Einstein disappointedly said: "The quanta really are a hopeless mess.” This paper will reveal the mystery: why photon concept can govern the scientific world until now, and give the evidences for the actual nature of light.
\end{abstract}

Keywords: Light, Wave, Vibration of Charged Particles, Photon, Wave-Particle Duality, Great Debates in Physics

\section{Introduction}

Physics is the base that every field of science depends on. In the past few centuries, the developments of physics were dependent on the alternate victory and defeat of three great debates. Recently, ostensibly, many new theories appear one after another; however, actually, many incurrect theories were developed or are being developed; because the century great debates have gone to an extreme error. In this paper we will tell you what an apple of discord of the three great debates is. And we will give evidences.

\section{The First Great Debate in Physics}

\subsection{Before 1704: Wave Property of Light Was Recognized Extensively}

Before 1704, most of scientists considered light as an ether wave. The representative scientists are Francesco Maria Grimaldi, Christiaan Huygens, Robert Hooke, and so on. At that time, Isaac Newton also contributed a lot to the wave nature of light. Their contributions are listed

as follows:

Francesco Maria Grimaldi (Italy, 1618-1663). In a book entitled Physico-Mathesis de lumine, coloribus et iride published posthumously, Grimald's observations of diffraction when he passed white light through small apertures were described. Grimaldi concluded that light is a fluid that exhibits wave-like motion.

Christiaan Huygens (Netherlands 1629-1695). At 1690, when he was 61 , he fully published his wave theory of light (in a communication to the Academie des Science in Paris, in his Traite de Lumiere in 1690). He suggested that light propagates as a disturbance (spherical pressure wave). He considered that each point of light wave can act as a secondary source of wavelets. One of the most important predictions of his theory was that light should propagate more slowly in a denser medium.

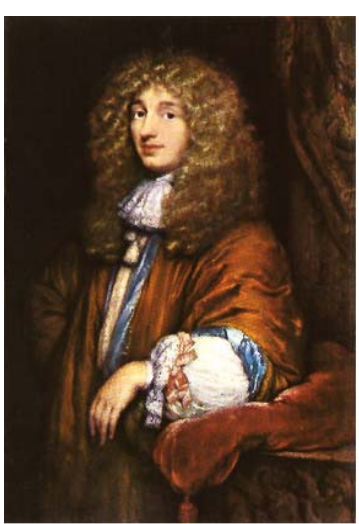

Christiaan Huygens 


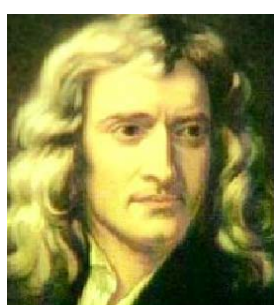

Sir Isaac Newton
Isaac Newton (England, 1643 1727). Newton's most remarkable observation about light wave was Newton's rings. Furthermore, Newton's earlier observation on the dispersion of sunlight as it passed through a prism is also an evidence of wave.

\subsection{Why Newton Proposed Corpuscular Concept?}

After Newton became the President of the Royal Society, the power of this top position and the glory made him arrogant. The adulation and acclamation made him abandon self-critical spirit. Newton mistook Hooke and Huygens as his enemies. In 1704, Newton proposed the incorrect concept of the corpuscular concept of light to destroy the wave theory of Hooke and Huygens. But this particle concept of light was incorrect; it could not explain the diffraction of light, so Newton had to explain diffraction of light using wave. They formed the waveparticle duality of Newton.

\subsection{Why did Newton's Wave-Particle Duality Govern the Scientific World for more than One Hundred Years?}

Newton proposed a particle concept of light in 1704, and died in 1727. Newton's incorrect wave-particle duality governed the physics world to 1818 . Why could the wrong wave-particle duality of Newton govern the physics world for near 100 years after Newton's death?

There is only one answer: the only causation was that at that time the reviewers (and the topic editors) came to fame due to their papers about the corpuscular concept (wave-particle duality) of light. For safeguarding their own honour and viscounty, they rejected all correct manuscripts.

\subsection{The Evidences of Wave in the 19th Century}

\subsubsection{Young Double Slit Interference Experiment}

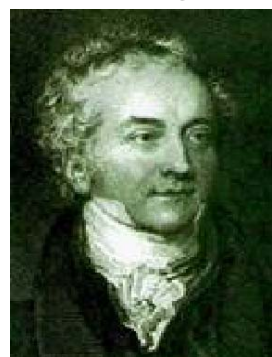

In 1801, Thomas Young (England, 1773 - 1829) finished his double slits interference experiment, which is one of the most important optical experiments in history. But his paper was rejected by the reviewers and editors for eighteen years, because it only can be explained well Thomas Young

\subsubsection{Poisson's Spot}

After many scientists, such as, Thomas Young and Augustin Jean Fresnel (France, 1788-1827), fought for a long time, the controversy of the Poisson's spot was held in France in 1818. When Fresnel reported his wave diffraction theory, SiméonDenis Poisson (France, 17811840) calculated the diffraction pattern of a disk using Fresnel's formula and got a result that a faint bright spot appeared at the centre of the disk. Poisson was one of the

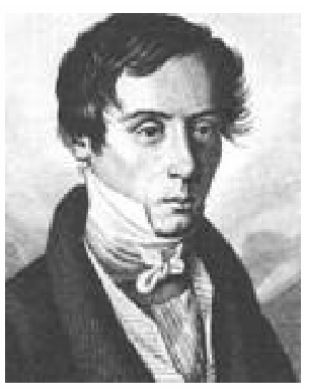

A. J. Fresne1

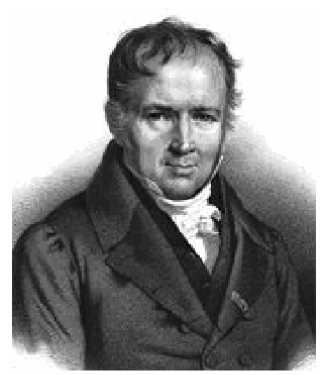

S. D. Poisson examiners of committee, and he was a believer in Newton's particle theory of light. Poisson said: "If you look at the shadow of a small circular object, the light bending from all sides would add up especially strongly in the middle. This means that in the middle of the shadow, there would be a faint bright spot. It is impossible.” The experiment was conducted at once, and the experiment validated the spot and verified that Fresnel's theory was indeed correct. Poisson was convinced of the wave theory. $\mathrm{He}$ presented the result to all the other judges to support Fresnel to be the winner of the contest. In order to memorize his dedication, this spot was named as Poisson's spot.

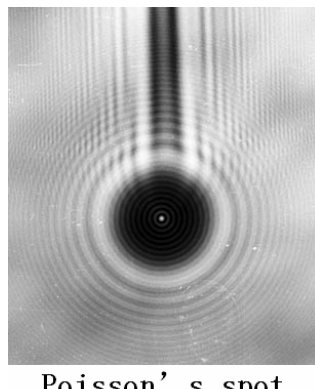

\subsubsection{Experimental Evidence about Velocity of Light in Air and Water}

Armand Fizeau and Jean Bernard Léon Foucault performed experiments to determine the velocity of light in air and water in 1849 and 1862 respectively. They found that the velocity of light in water was much slower than that in air. It is exactly the same as Huygens's predicttions of his wave theory. This became one of the important evidences of the wave concept of light, because particle concept predicted that light in air was slower than that in water.

\subsubsection{Electromagnetic Wave}

When James Clerk Maxwell (Scotland, 1831-1879) fin- 


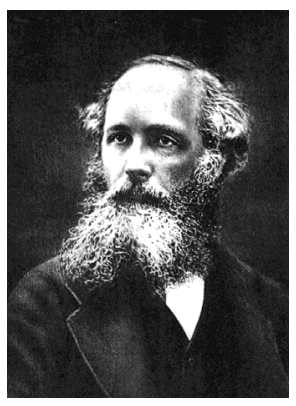

ished the research of his Maxwell equations, he calculated the speed of electromagnetic waves and found that it was $300,000 \mathrm{~km} / \mathrm{sec}$, which was the same of light. This forced Maxwell to ponder about the nature of light: it must be true that the light is in fact an electromagnetic wave! Maxwell concluded that light is a form of electromagnetic wave. Maxwell wrote: "This velocity is so nearly that of light that it seems we have strong reason to conclude that light itself (including radiant heat and other radiations) is an electromagnetic disturbance in the form of waves propagated through the electromagnetic field according to electromagnetic laws."

\section{The Second Great Debate in Physics}

\subsection{Black-Body Radiation}

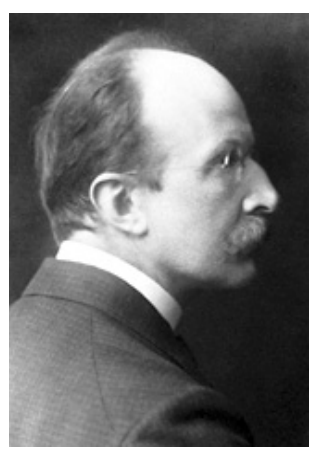

For the explanation the spectra of black-body radiation, Max Karl Ernst Ludwig Planck (Germany, 1858 - 1947) firstly proposed that emission of black-body was energy quantization with value of $\hbar \omega$, and he introduced the Planck constant $\hbar$. [1] It was the beginning of Quantum Physics, and Planck was recognized as the originator of Quantum Physics, so, in 1918, he got the Nobel Prize in Physics.

However, Planck believed that the origin of his $\hbar \omega$ had not been discovered; and he himself struggled for it throughout his life. Until his death, he never accepted his $\hbar \omega$ as a photon.

\subsection{How was the Wrong Concept of Photon Proposed and Developed?}

\subsubsection{The Proposition of Photon was a Sudden Whim} In 1905, Albert Einstein (Germany, 1879-1955) was busy on writing his $\mathrm{PhD}$ thesis. In March, a month before he finished his $\mathrm{PhD}$ thesis, he had a sudden whim that photoelectric effect could be explained by a hypothesis of quanta (photon). So he spent a few days in writing a paper and submitted it in March 1905 (It was published in June 1905). In this hypothesis for explaining photoelectric effect, he only considered the energy conservation and made use of Planck's $\hbar \omega$ [1] to propose a quanta concept of light [2]. The year 1905 is the busiest time of Einstein. He did a lot of great works in that year. He was really too busy in 1905 , he did not thoroughly consider the concept of photon, which caused him regret throughout his life.

Planck wrote a letter to Einstein in 1907 to tell him that $\hbar \omega$ was not the property of light in propagation; it only appeared in the interaction with charged particles. Light in propagation could only be described by Maxwell equations. Since that, Einstein began to doubt the concept of photon. This made Einstein uneasy throughout his life, which Einstein hoped to resolve, but could not. So he said: "I spent all my life trying to understand what a photon is, and haven't understood it by now" [3]. And finally, he said: "The quanta really are a hopeless mess" [3].

\subsubsection{Millikan's Experiment Misguided the Nobel Prize Committee and Afterward these Nobel Prizes Misguided the Whole Scientific World}

Before 1916, nobody considered the concept of photons as correct, and Planck strongly opposed the concept of photon. However, in 1916, R. A. Millikan reported an experimental result, which also considered the energy conservation only to prove the formula of photoelectric effect with mechanism of the collision between photon and free electron [4]. In R. A. Millikan's experiment, he did not consider the momentum conservation law. Their hypothesis contravenes the momentum conservation law, and the $0.5 \%$ precision of Millikan's experiment demonstrates that photoelectric effect does not result from the collision between photons and free electrons. In another paper of this series papers, we will give the evidences in detail for the actual mechanism of photoelectric effect.

The worst thing is as follows: Einstein proposed theory of relativity, stimulation theory of light, the gravitational waves, and so on. So, everybody thought that Einstein should have deserved to win several Nobel prizes. In 1921 committee of the Nobel Prize decided to award Einstein a prize. Unfortunately, affected by Millikan's experiment, the opinion of

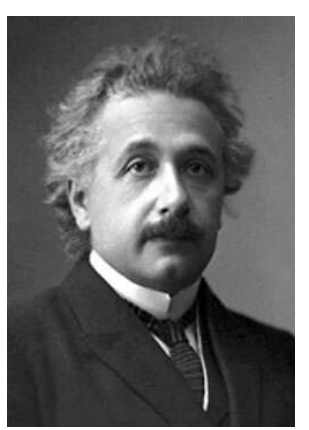
some committee members was that only quanta had experimental proof, so in Einstein's Nobel Prize only the photon was mentioned, although all members of the Nobel Prize committee thought that Einstein deserved to win Nobel prize. Because Einstein is a very famous scientist and his Nobel Prize only mentioned photons, and so many Nobel Prizes in physics awarded were related to the concept of photon. They made the concept of photon accepted universally and misguided the whole scientific world. 


\subsubsection{Nobel Prize Misguided Compton to Get a Wrong Conclusion}

In 1923, under the misguiding above, Compton although knew that there were four puzzlements and quandaries explaining photonic collisions (such as Compton's scattering results of various elements were undoubtedly evidences that prove that the collision between photon and nucleus is inexistent and untenable), he finally still explained his experimental result as a photonic collision [5]. Because it considered both the energy and momentum conservation law, Compton scattering was misunderstood as the most convincing experimental evidence for photon concept. The quandaries that Compton met and the actual mechanism will be discussed in detail in another paper of this series papers.

\subsection{Who Give the Name of "Photon" and what is it in Scientists' Mind?}

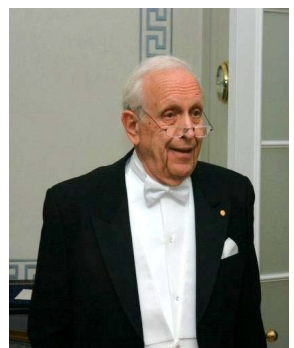

The word "photon" was proposed by Gilbert Newton Lewis (18751946, American) in 1926 [6].

In fact, in the mind of the most famous scientists on quantum optics: photon is an inexistent entity. Such as the Nobel Prize (Quantum Optics) laureate Roy Jay Glauber (born in 1925) said: "A photon is what a photo-detector detects." "A photon is where the photo-detector detects it" [7]. This implies that photon is the characteristics of photo-detector rather than traveling light itself.

\subsection{Evidences that Prove the Concept of Photons Is Wrong}

In this series papers, we give 9 evidences (each one will be one paper in this series papers). They directly prove that the concept of photons is wrong. They are summed up as follows:

1) Four evidences (lithium does not have a P band, photonic explanation contravenes the impartibility of a photon, all measurements depend on wave property, without recoil force of photonic collision) prove that explaining Compton scattering using photons is wrong. Our results show Compton scattering is actually the Recoil-Doppler- Rayleigh scattering of free electrons.

2) The photonic explanation of photoelectric effect contravenes the law of conservation of momentum. Our experimental results demonstrate that the photoelectric effect is an effect of a wave induced dipole of a surface electron. The frequency dependent quantized property of this light induced dipole perfectly explains all phenom- ena of the photoelectric effect.

3) The mainstream photon model experiment actually detects the emissions at different times and the superposition of wave trains from different atoms. From their references [8-10], we find that they only record one count of emissive light energy $\hbar \omega$ of the superposition of many wave trains from many atoms (every pulse, $10^{9}$ atoms emited light trains, and every detector detected the superposition of wave trains from about $10^{5}$ atoms, according to their experimental systems). The temporal response time of their photomultiplier is about 2 ns. It means that their photomultiplier is no possible to distinguish the light trains from different atom within a pulse. Furthermore, for emissions at the same time, the uptransition times of electronic vibration states also make the response times of the two detectors different, due to the different micro-distribution the superposition of wave trains and the different micro- structure of detector.

4) Taylor's feeble light interference experiment can be explained well with the combination of wave diffraction and the different up-transition time of vibration states of different molecule, while photons can not explain it.

5) We design an experiment, in which light is detected at constant power and different beam widths. The measured results depend on wave density rather than the number of photons. This proves that light should be explained by a wave.

6) Combining experiment, classical theory and quantum theory, our results demonstrate that quantized energy $\hbar \omega$ in black-body radiation comes every time a single hot electron emits a light wave train with energy of $\hbar \omega$. Namely, quantized energy $\hbar \omega$ in black-body radiation comes from particle property of electron.

7) Raman scattering is the combination of light wave induced quantized dipole and molecular intrinsic vibration between nucleuses (include rotation), rather than photons emits from the virtual levels. Or say, the virtual level is a real level of light induced vibration dipole.

8) According to quantum mechanics, the spontaneous emission of the excited atomic stationary state is impossible in absent of perturbation. Using vibration dipole as a bridge process, spontaneous emission can be explained.

9) In the past, under the misguidance of the photon, the mechanism for pair production is confusing. Electron-positron pair annihilation with the production of $\gamma$ rays was predicted by Dirac. It was almost immediately observed, and it has since become possibly the most prolific field of research in the active domain of particle physics (more than a half excellent results and papers in particle physics came from this field). On the other hand, electron-positron pair production by light-light collisions was predicted by Breit-Wheeler. Because some part of Quantum Electro-Dynamics was developed from it, 
Quantum Electro-Dynamics predicts that it is possible. Many expensive work have been done for it, it wasted a lot of money and time of human being to prove it, but never success. According to our theory, Dirac's Electron-positron pair annihilation process is correct, while, Breit-Wheeler's Electron-positron pair by light-light collisions is wrong. Our theory will stop the wrong experiments to prove Breit-Wheeler's prediction and the waste of money and time of human being. According to our theory, we propose the law of spontaneous transform of matter, which unifies the mechanism of all pair creation methods.

Because the theoretical and experimental results and their discussions are very long, every one of the evidences above will be written a long paper. So every single evidences above will be detailed respectively in one of this series papers (Namely, 9 evidences above will appear in nine papers).

\section{Photon concept worsens Einstein-Bohr Great Debate}

In the Einstein-Bohr great debate, the opinions of Einstein and Schrödinger are: 1) Materialistic objective reality is independent of the people's will. 2) They insisted on the existence of causality. 3) They insisted on the locality and denied the non-locality. They recognised that quantum mechanics can give probability without asking for the parameters in micro-scale, which is great progress. However, they pointed out that the shortcomings of quantum mechanics lead to the illogical quantum entanglements with spooky remote effects. However, recently, under the help of wrong photon concept, some bodies called themselves the "mainstream scientists" and called Einstein and Schrödinger the representative of "Nonmainstream, anti-mainstream, or wrong researchers”. It makes many people misunderstand and mistake Einstein and Schrödinger as incorrect scientist.

How photon concept did worsen Einstein-Bohr great debate? It needs a long paper to describe it in detail, which will be reported in another paper of this series papers. In the following paragraphs, we only give a short summery.

\subsection{Well Known Arguments in Einstein-Bohr Great Debate}

There are three well-known arguments appeared in 1927, 1930, and 1935. The first and second arguments were based on the wrong photon concept. However, because the discussed topic related to the wrong concept of photon, wrong concept made arguments far apart from physical principle, wrong concept can not give correct result, and made Einstein's original idea unable to be comprehended by Bohr. And Bohr used the topic of photon to easily make Einstein speechless. The number of majority of Copenhagen group won the argument, but could not convince their opponents. It moved the debate further apart from the correct direction.

The third argument is the most important one. In these two decades, the wrong concept of photon also makes people mistakenly think Einstein and Schrödinger seem wrong in the third argument. This mistake makes many physicists waste a lot of money and time of human being to develop wrong theory and conduct wrong experiments. It will be discussed curtly in the following sections.

\subsection{The Third Argument in Einstein-Bohr Great Debate}

\subsubsection{The EPR Paper and Schrödinger's Cat}

In 1935, the viewpoints of Einstein's EPR paper [11] and Schrödinger's cat [12] are as follows: Quantum Mechanics had a shortcoming that will give a confusing state and has spooky remote effect. So quantum mechanics should be further improved. Einstein and Schrödinger give this spooky remote effect a name of quantum entanglement.

Five months later, Bohr published a response paper, which was very difficult to read. Bohr emphasized that no matter how far the distance of the two particles was, they could interact with each other. The rest part of Bohr's paper gave some irrelevant answers.

Before the publication of this EPR paper, Bohr uncompromisingly argued against the non-locality. However, after the publication of this EPR paper, he never rejected non-locality, and encouraged his students and follower to develop it.

\subsubsection{A Turning Point of Photonic Quantum Entanglement}

Before 1980, a lot of people studied this topic, and no conclusion was accepted extensively. In 1981-1982, there are three experimental papers as the experimental evidences for quantum entanglement published in Physical Review Letters [8-10]. It became a turning point. Actually, they have no novel content in comparison with their references, [13-15] expect for the adoption of high density laser pumping. For every body's intuition, to detect a single photon without disturbing with each other, it needs weak light, because the weaker the light is, the longer the temporal interval will be. Only when the temporal interval larger than the response time of the detector, you can detect single photon without disturbing with each other. Why the high density laser worked? The answer is that their experiment instruments were incapable to detect 
fluorescence from a single atom at all. Their detected results are the superposition of many light wave trains from many calcium atoms.

1) Both the sensitivity and temporal property of photomultiplier prove that it can not detect fluorescence from a single atom.

According to the parameters in the literature, we sum up their parameters and make estimation as follows: 1) One record was detected from several hundred pulses. 2) In one pulse, fluorescence of $7.16 \times 10^{7}$ (at least $10^{5}$ ) calcium atoms enters one photomultiplier. 3) The temporal response time of photomultiplier is about 2 ns. 4) The fluorescent life time is much longer than the temporal response time of photomultiplier. It means that their photomultiplier is no possible to distinguish the light trains from different atom within a pulse. Or say, this system is absolutely impossible to detect light emitted from one atom. Namely, their photomultiplier can not distinguish the fluorescence train from a single atom, among fluorescence train from so many atoms.

In one word, they used laser pumping, and they detected the superposition of many wave trains from many calcium atoms in every pulse, so the detected results were stable.

\section{2) The actual physics in these experiments}

These experiments only detected the superposition of many light wave trains from a lot of atoms, rather than a fluorescence pairs from a single calcium atom. In the following, two examples are described.

\section{Example one:}

At intense laser pumping, population inversion of partial calcium atoms is possible to appear. It will appear the local (centre of the Gaussian laser beam for example) coherent or partial coherent radiation (lasing, super-radiance or super- fluorescence). Namely, light that enters the photomultiplier is the superposition of coherent emission and fluorescent wave trains of random polarization from many atoms.

If the polarization of coherent radiation is in the direction $\mathrm{Y}$ and its light intensity is $I_{s}$; in a direction $A$, the light intensity $\left(I_{A}\right)$ is the sum of projection of coherent radiation in direction $\mathrm{A}$ and the random fluorescence $\left(I_{R}\right)$. The projection is function of angle's cosine, so we get the light intensity in direction $\mathrm{A}: I_{A}=I_{S} \cos \theta+I_{R}$. The coincidence rate between directions $\mathrm{Y}$ and $\mathrm{A}$ depends on $I_{A}$. So the coincidence rate as a function of the relative polarizer orientation depends on $I_{A}=I_{S} \cos \theta+I_{R}$. Namely the coincidence rate as a function of the relative polarizer orientation is a function of angle's cosine adding a back ground, which comes from the random fluorescence. The experimental result in these three papers is a similar cosine function adding a back ground; it is exactly the same as our analysis.

\section{Example two:}

At weak excitation (previous experiment of the references of these three papers), population inversion does not appear. Only many calcium atoms simultaneously emit wave trains with the same polarization can be detected. The probability of that many calcium atoms simultaneously emit wave trains with the same polarization is small, so it was difficult to get a record in those experiment before 1981 .

If the same polarization direction of simultaneously emission is in direction $\mathrm{Y}$, which light intensity is signed by $I_{y}$; and that the direction of polarizer in front of the second photomultiplier is in direction A, light intensity of in direction $\mathrm{A}\left(I_{A}\right)$ should be the projection of $I_{y}$ in direction A, plus the random component $\left(I_{R}\right)$. When light intensity is higher than the threshold of the photomultiplier, the coincidence signal will be recorded. So the coincidence rate as a function of the relative polarizer orientation will be the same of $I_{A}=I_{y} \cos \theta+I_{R}$. It is a cosine function, adding a background of the random component. It is consistent with the experimental results.

The two examples above demonstrate that the experimental results above are the result of supposition of light wave trains. Of course, the examples above are the simplified analyses. The precise method is a statistical method.

\subsubsection{What Is Photonic Teleportation Experimental Evidence Wrong?}

Another and the most important experimental evidence for non-locality and quantum entanglement is a paper published in Nature.[16] There are five problems prove that their evidence is untenable. In the following, we sum up two of them as follows:

1) As described by authors (line 15-16 of second column in page 578), all of their experimental results indicate "that photon 3 is polarized along the direction of photon 1, confirming teleportation”. In fact, the authors know the polarization of photon 1 because they inset a polarizer in it, and they have detected polarization of photon 3 directly. This is the real evidence. The authors have measured it. Why don't the authors dare tell readers about this real direct evidence?

2) The authors gave Fig. 4 and Fig. 5 as their experimental evidences, but using wave without entanglement can give these results. Furthermore, Fig. 4 and Fig. 5 are not same as Fig. 3 (the theoretical result of teleportation). The peak value in their Fig. 3a should be zero, but in Fig. 4 and Fig. 5 is not zero, which is the same as the explanation of wave. The detail is too long; so it will be detail in another paper.

\subsubsection{The Recent Status of Quantum Entanglement}

The recent study of quantum entanglement can be classi- 
fied into the following three categories:

\section{1) Photonic quantum entanglement}

Quantum Mechanics had a shortcoming that will give a confusing state and has spooky remote effect. So using Quantum Mechanics, it is easily to get Photonic quantum entanglement. However, we have proved that all of their experimental evidences are incorrect.

2) Quantum entanglement of atom following photonic method

Recently, some of theoretical researchers reported some papers about quantum entanglement of atom following the photonic method. They imagine that atoms interact and entangle with each other, and then these entangled atoms are separated with entanglement to achieve spooky remote effects. However, there is no convincing experimental evidence to support it.

3) "Quantum entanglement of atom" with interacttion of field and without spooky remote effect

Many theoretical and experimental researchers are conducting these researches. It is totally different from photonic quantum entanglement. Its interaction is generated by the field of high Q cavity. Although most of the researchers used the same description method and test method of photonic quantum entanglement to treat these experiments, it actually differs from photonic quantum entanglement and the quantum entanglement proposed by Einstein and Schrödinger, its physics is correct. When photonic quantum entanglement withers away, it will still exist with another name.

Summing up the results above in Section 4, we can conclude that 1) the experimental evidences of photonic quantum entanglement actually is the results of the superposition of many wave trains from many calcium atoms in every pulse, rather than a single photon; 2) in Einstein-Bohr great debate, the viewpoints of Einstein and Schrödinger are correct.

\section{References}

[1] M. Planck, “The Theory of Heat Radiation,” Translation by Morton Masius, P. Blakiston's Son \& CO., Philadelphia, 1914.

[2] A. Einstein, "Concerning a Heuristic Point of View toward the Emission and Transformation of Light," Annals of Physics, Vol. 17, 1905, pp. 132-148. doi:10.1002/andp.19053220607

[3] M. Born, "The Born-Einstein letters 1916-1955, Friendship, Politics and Physics in Uncertain Times,” By Max
Born, Macmillan, New York, 1971, 2005.

[4] R. A. Millikan, “A Direct Photoelectric Determination of Planck's 'h'," Physical Review, Vol. 7, No. 3, 1916, pp. 355-388. doi:10.1103/PhysRev.7.355

[5] A. H. Compton, "A Quantum Theory of the Scattering of X-Rays by Light Elements,” Physical Review, Vol. 21, 1923, pp. 483-498. doi:10.1103/PhysRev.21.483

[6] G. N. Lewis, “The Conservation of Photons,” Nature, Vol. 118, No. 2981, 1926, pp. 874-875. doi:10.1038/118874a0

[7] “The Nature of Light, What is a Photon?” P39, Edited by Chandrasekhar Roychoudhuri A. F. Kracklauer Katherine Creath, CRC Press, Taylor \& Francis Group, Boca Raton London New York, 2008.

[8] A. Aspect, P. Grangier and G. Roger, "Experimental Tests of Realistic Local Theories via Bell's Theorem," Physical Review Letters, Vol. 47, No. 7, 1981, pp. 460-463. doi:10.1103/PhysRevLett.47.460

[9] A. Aspect, P. Grangier and G. Roger, "Experimental Realization of Einstaein-Rosen-Bohm Gedankenexperiment: A New Violation of Bell's Inequalities,” Physical Review Letters, Vol. 49, No. 2, 1982, pp. 91-94. doi:10.1103/PhysRevLett.49.91

[10] A. Aspect, J. Dalibard and G. Roger, "Experimental Tests of Bell's Inequalities Using Time-Varying Analyzers," Physical Review Letters, Vol. 49, No. 25, 1982, pp. 1804-1807. doi:10.1103/PhysRevLett.49.1804

[11] A. Einstein, B. Podolsky and N. Rosen, "Can Quantum-Mechanical Description of Physical Reality Be Considered Complete?” Physical Review, Vol. 47, No. 10, 1935, pp. 777-780. doi:10.1103/PhysRev.47.777

[12] E. Schrödinger, "Die Gegenwartige Situation in der Quantenmechanik (The Present Situation in Quantum Mechanics)," Naturwissenschaften, Vol. 23, 1935, pp. 807-812, 823-828, 844-849.

[13] S. J. Freedman and J. F. Glauser, "Experimental Test of Hidden-Variable,” Physical Review Letters, Vol. 28, No. 14, 1972, pp. 938-941. doi:10.1103/PhysRevLett.28.938

[14] J. F. Glauser, "Experimental Investigation of Polarization Correlation Anomaly,” Physical Review Letters, Vol. 36, No. 21, 1976, pp. 1223-1226. doi:10.1103/PhysRevLett.36.1223

[15] E. S. Fry and C. Randall, "Thompson, Experimental Test of Hidden-Variable Theories,” Physical Review Letters, Vol. 36, 1976, pp. 465-469.

[16] D. Bouwmeester, J.-W. Pan, K. Mattle, M. Eibl, H. Weinfurter and A. Zeilinger, "Experimental Quantum Teleportation,” Nature, Vol. 390, No. 6660, 1997, pp. 575-579. doi:10.1038/37539 